\title{
ATTENUATION BEHAVIOR OF SOLID DENSE RANDOM MEDIA AT MICROWAVE FREQUENCIES
}

\author{
Nadimi S. A., Bredow J.W., Fung A. K. \\ Wave Scattering Research Center \\ Department of Electrical Engineering, Box 19016 \\ University of Texas at Arlington Texas 76019, USA
}

\begin{abstract}
To better understand scattering from nontenuous dense random media such as sea ice and snow, attenuation measurements have been performed on two different types of random media with ka values ranging from 0.5 to 0.7 , and 1.5 to 2.1 . The following paper describes, briefly, experimental results from wave propagation in plane slabs of finite thickness composed of: (1) a random distribution of identical, finite scatterers; and (2) a random distribution of scatterers with narrow size distribution. The aim of this paper is to present the observed behavior in terms of attenuation versus volume fraction, and, additionally, the behavior of attenuation versus frequency. Results presented in this paper are compared to the behavior reported by some earlier experiments where the medium properties are different.
\end{abstract}

\section{INTRODUCTION}

The understanding of the behavior of the attenuation constant of random media, as a function of volume fractions and/or frequency (ka), has gained increasing attention due to its importance in many practical problems, such as wave propagation in the atmosphere, sea ice, and snow.

When a plane wave with initial intensity $I_{0}$ propagates through a random layer of particles of thickness $L$, it experiences attenuation such that $I_{c}$, the intensity after encountering the medium, is [3]:

$$
\frac{I_{c}}{I_{o}}=\exp (-\alpha L)
$$

$\alpha$ is the attenuation constant measured in $\mathrm{Np} / \mathrm{m}$ and is given by:

$$
\alpha=-\frac{10\left[\log \left(\frac{I_{c}}{I_{o}}\right)\right]}{4.343 \mathrm{~L}}
$$

Strictly speaking, for $\alpha$ to be characteristic of a given medium, i.e., independent of the medium dimensions, the plane wave must be propagating through a slab of infinite extent. However, accurate measurements of $\alpha$ can be obtained in the lab if one is careful that the wave incident on the target is approximately a plane wave; and that the target fully occupies the beam. To measure the attenuation through a slab of scatterers with a given thickness $L$ the far field requirements of the antennas must be met. The general far field requirement is $2 D^{2} / \lambda_{0}$ where $D$ is the maximum aperture dimension, and $\lambda_{0}$ is the free space wavelength.

\section{SYSTEM DESCRIPTION}

All of the measurements were performed using the anechoic chamber of The Wave Scattering Research Center at The University of Texas at Arlington. A simplified configuration of the attenuation measurement system used for this study is shown in Figure 1. The main component of this system is the HP 8510A network analyzer. The $8510 \mathrm{~A}$ network analyzer was chosen due to its accuracy, wide dynamic range, good phase stability, high speed data acquisition /computation, and its capability to handle a large number of measurement points.

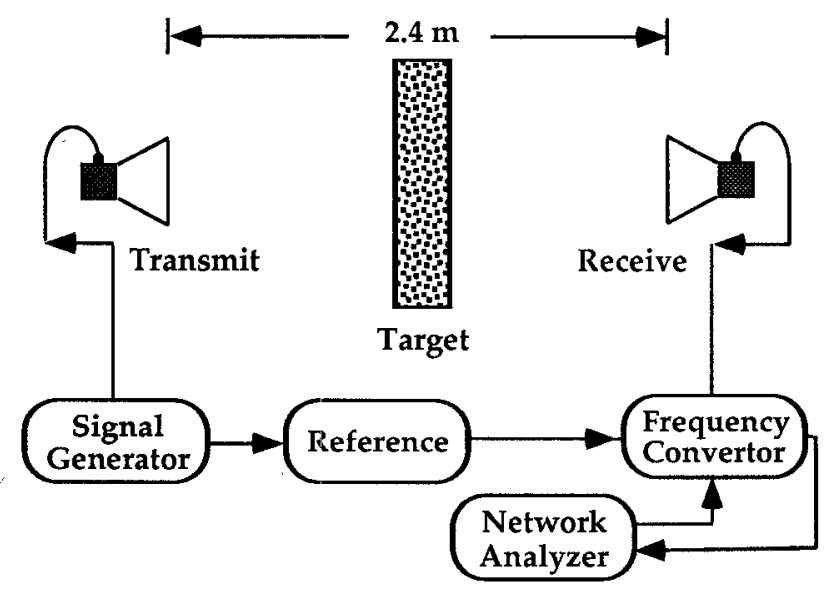

Figure 1. Simplified Configuration of the Measurement Set

The other components of this system consist of a frequency synthesized source, variable attenuator, broadband directional coupler, frequency convertor, microwave amplifier, and transmitter and receiver antennas. The transmit and receive antennas are 
dual-polarized conical horns with frequency range from 2 to 18 $\mathrm{GHz}$. The overall operating bandwidth of the system is from 2 to $18 \mathrm{GHz}$ as well.

The transmitter antenna was mounted on a fixed stand, while the receiver antenna was mounted on a tripod to allow flexibility for antenna pattern measurements and boresighting purposes. The antennas were positioned inside the anechoic chamber at 1.4 meters above the ground, and to satisfy the plane wave criteria, they were placed 2.4 meters apart (at far-field with respect to each other). To study the radiation pattern of the antennas, the $3 \mathrm{~dB}$ beamwidths of the antennas were measured at $1.2 \mathrm{~m}$, to be $20^{\circ}$ and $12^{\circ}$, at 9.5 and $14.0 \mathrm{GHz}$ respectively, illuminating an area of 25 to $42 \mathrm{~cm}$. A target stand with height of 1.1 meters was made of styrofoam with a dielectric constant of 1.03 and was placed inside the anechoic chamber at the center between the two antennas.

\section{TARGET DESCRIPTION}

For this experiment two sets of targets (thickness approximately $22 \mathrm{~cm}$ diameter approximately $63.5 \mathrm{~cm}$ ) were constructed: the first set consisted of $14.3 \mathrm{~mm}$ (i.e., $\mathrm{ka}=1.5$ to 2.1 ) diameter marbles with dielectric constant of 6.3 imbedded within a slab of polystyrene; and the second set consisted of rocks with dielectric constant of 5 imbedded within a slab of polyethylene foam. The rocks were selected from two sifts consisting of sizes ranging from 4.0 to $5.6 \mathrm{~mm}$ (i.e., $\mathrm{ka}=0.5$ to 0.7 using $\mathrm{a}=4.8 \mathrm{~mm}$ ). The first set of targets, consisting of $4,8,16$, 24 , and 36 percent volume fractions, was constructed by randomly inserting the scatterers into foam layers each having thickness of $3.2 \mathrm{~cm}$. The second set, consisting of 16 , and 28 percent volume fractions was constructed by randomly spreading the scatterers on foam layers each having thickness of 0.79 $\mathrm{mm}$. Two additional targets having dimensions identical to those of the scatterer targets but less the scatterers, were constructed from the same materials to account for the foam media (background).

\section{MEASUREMENT TECHNIQUES}

The first step in the measurement sequence was to study the behavior of the target stand, and the media in which the scatterers were embedded. No behavior that could have any significant effect on the measurements was noticed. Once this was achieved, measurements of the scatterer targets were done using the following procedure: (1) the empty chamber, including the target stand and the medium of foam without the scatterers was measured and the data was saved to be used later for data processing purposes; and (2) the target with scatterers was measured. For each target, 96 samples were obtained so average out fading effects. Data was then processed to obtain the attenuation constant for each target.

\section{DISCUSSION}

The results for all volume fractions indicate a consistent increase in attenuation as frequency is increased; this behavior is shown in Figures 2 and 3. In addition, the behavior of attenuation versus volume fraction for both sets of targets is shown in Figures 4 and 5. Figures 2-5 show, that with respect to both frequency and a given volume fraction, the increase of the attenuation is faster for the smaller scatterers than for larger scatterers. Observe that Figures 4 and 5 show a consistent monotonic increase in attenuation as the volume fraction is increased. This behavior is not the same as the behavior reported elsewhere [1], and [2] of lightwave attenuation through a medium consisting of teflon particles suspended in liquid (with $\mathrm{ka}=0.6$ and 0.99 ). For those, attenuation decreased for the smaller particles, as the volume fraction was increased above $12 \%$. Gibbs and Fung, [1], attribute this behavior to a decrease in the effective absorption of the dispersion. Earlier, Ishimaru and Kuga, [2] attributed this decrease to particle correlation.

Results indicate that a decrease in attenuation did not occur after the volume fraction exceeded $12 \%$. However, this does not exclude the possibility that attenuation may decrease at a larger volume fraction (greater than 28 percent). Experimental and theoretical research are under way to further analyze this behavior through construction and modeling of additional volume fractions consisting of smaller scatterers.

\section{ACKNOWLEDGMENT}

This study was supported by NASA grant NAGW 2344.

\section{REFERENCES}

1. Gibbs, D.P. and Fung, A. K., "Measurement of optical transmission and backscatter from a dense distribution of parti cles," Proc. IGRSS `90 Symposium, vol. II, pp. 1029-1032.

2. Ishimaru, A. and Y. Kuga, "Attenuation constant of a coherent field in a dense distribution of particles," J. Opt. Soc. Am., vol. 72, pp. 1317-1320, Oct. 1982.

3. Ishimaru, A., "Wave propagation and Scattering in Random Media" (Academic, New York, 1978). 


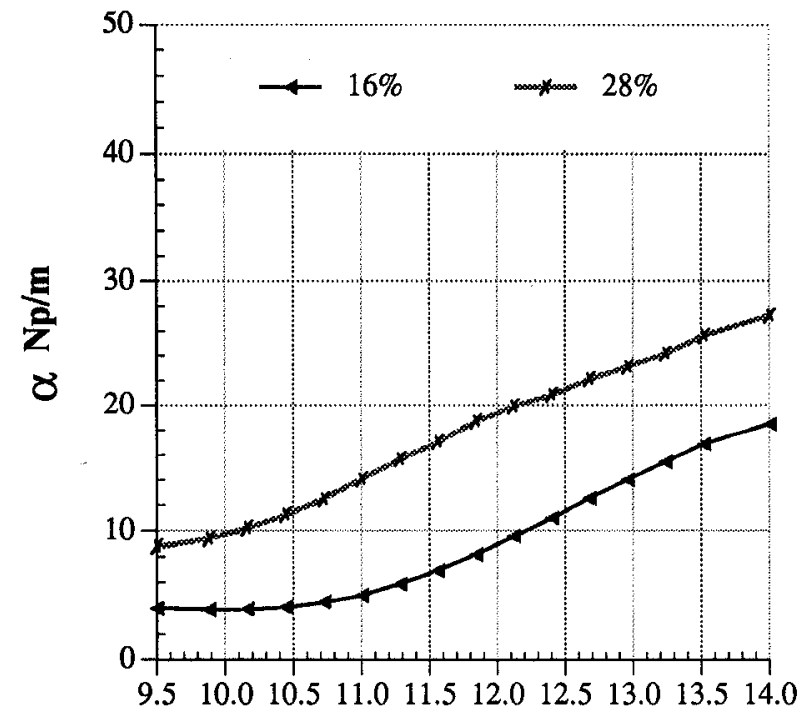

Frequency (GHz)

Figure 2. Attenuation constant vs, frequency for targets with ka values between 0.5 to 0.7

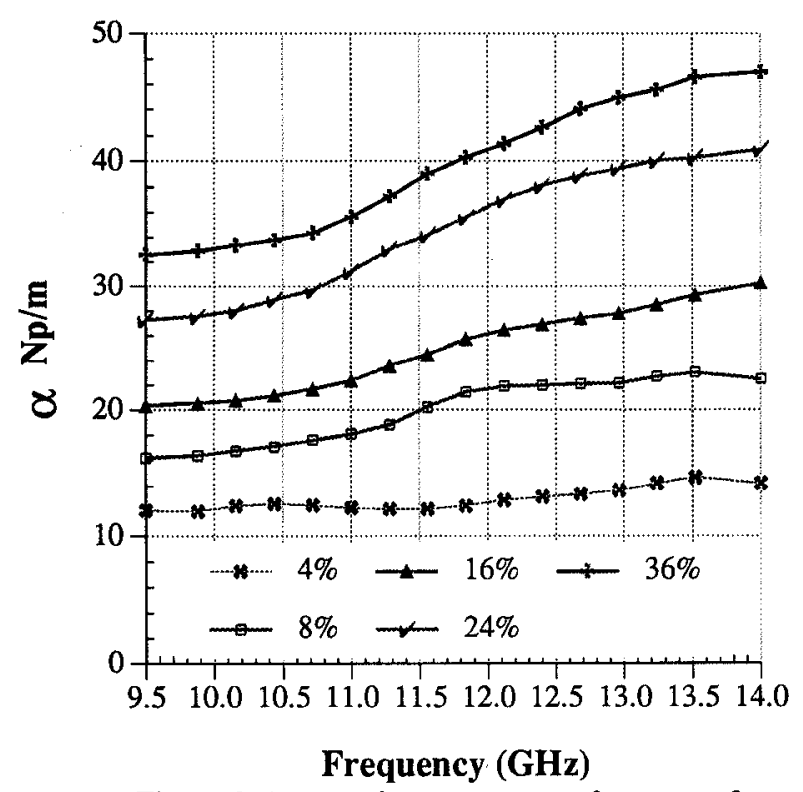

Figure 3. Attenuation constant vs. frequency for targets with ka values between 1.5 to 2.1

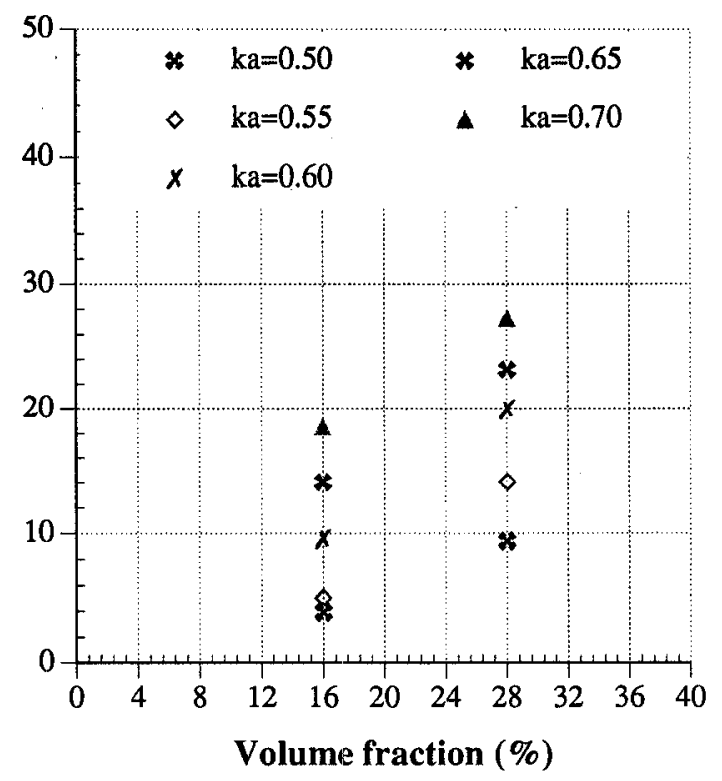

Figure 4. Attenuation constant vs. Volume fraction for targets with ka values between 0.5 to 0.7

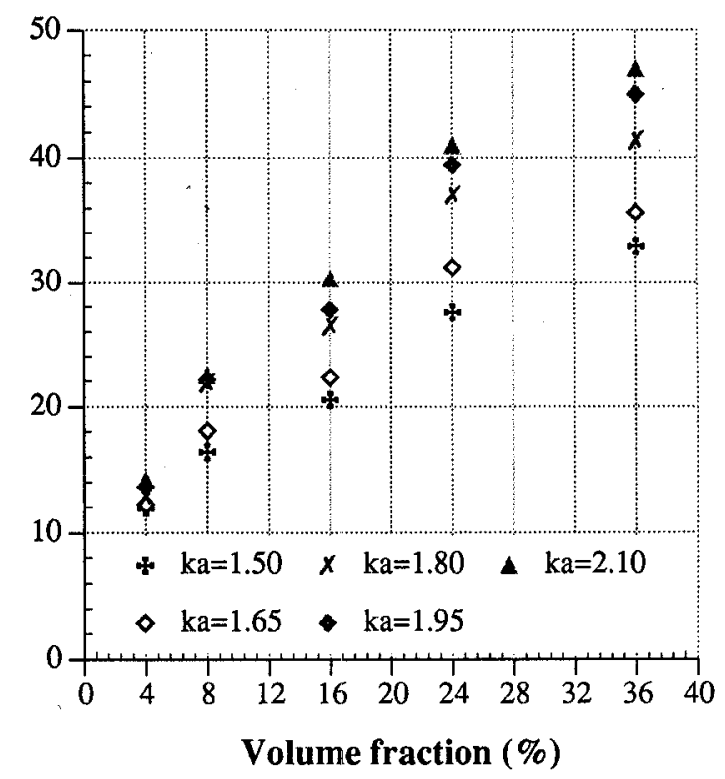

Figure 5. Attenuation constant vs. Volume fraction for targets with ka values between 1.5 to 2.1 